\title{
Dynamics of PEO in Blends with PMMA: Study of the Effects of Blend Composition via Quasi-Elastic Neutron Scattering
}

\author{
Victoria García Sakai ${ }^{\dagger}$ \\ Department of Chemical Engineering, The Pennsylvania State University, \\ University Park, Pennsylvania 16802
}

\author{
Janna K. Maranas* \\ Department of Chemical Engineering, The Pennsylvania State University, \\ University Park, Pennsylvania 16802
}

\section{Inmaculada Peral ${ }^{\ddagger}$}

NIST Center for Neutron Research, National Institute of Standards \& Technology, Gaithersburg, Maryland 20899, and Department of Materials Science and Engineering, University of Maryland, College Park, Maryland 20742

\author{
John R. D. Copley \\ NIST Center for Neutron Research, National Institute of Standards \& Technology, \\ Gaithersburg, Maryland 20899
}

Received July 5, 2007; Revised Manuscript Received February 19, 2008

\begin{abstract}
We address the dynamic behavior of poly(ethylene oxide) [PEO] in miscible blends with poly(methyl methacrylate) [PMMA] by using quasi-elastic neutron scattering [QENS] with isotopic labeling. The data reveal two dynamic processes in the picosecond-nanosecond time scales: a slow process that is consistent with previous measurements of the segmental relaxation and a composition independent fast process occurring on the picosecond time scale. The composition dependence of the slow process differs from previous measurements, particularly at low PEO content. The fast process is similar to the fast process observed in pure polymers and is insensitive to blending with PMMA. Relaxation times extracted from Kolraush-Williams-Watts [KWW] fits to the data are used to test the applicability of the chain connectivity and coupling models as a function of spatial scale. Both models describe slow process relaxation times within a small range of spatial scales near the Kuhn length of PEO. The effective concentration, when obtained as a fit parameter in chain connectivity model fits, is not a constant, but it decreases with increasing spatial scale.
\end{abstract}

\section{Introduction}

Poly(ethylene oxide) $[\mathrm{PEO}]$ and poly(methyl methacrylate) [PMMA] form miscible amorphous blends at PEO concentrations not greater than $30 \%$ by weight. This system is unique among other blends for which dynamics have been addressed because of a number of unusual characteristics. PEO/PMMA blends have a negligible $\chi$ parameter, owing to the minimal thermodynamic interactions between the two polymers. ${ }^{1,2}$ The difference in glass transition temperatures $\left[T_{\mathrm{g}}\right.$ 's $\mathrm{s}$ between $\mathrm{PEO}$ and PMMA is significant $(\sim 180 \mathrm{~K})$, with PEO having the low $T_{\mathrm{g}}$ and thus being the more mobile component. Two dynamic responses are observed through a variety of experimental techniques, ${ }^{3-12}$ and two glass transitions have recently been reported. ${ }^{58}$ If characteristic relaxation times are compared at a common temperature, the mobility of PMMA increases with blending while that of PEO decreases. Although the difference in the blend is smaller than that between the pure components, component mobilities remain widely separated. A study ${ }^{9}$ using ${ }^{2} \mathrm{H}$ nuclear magnetic resonance [NMR] suggests that segmental relaxation times of PEO may be up to 12 orders of magnitude

\footnotetext{
* To whom correspondence should be addressed. E-mail: jmaranas@ engr.psu.edu.

${ }^{\ddagger}$ Present address: ISIS Facility, The Rutherford Appleton Laboratory, Chilton, Didcot, OX 11 0QX, U.K.

* Present address: Institut de Ciencia de Materials de Barcelona (CSIC), Campus de la Universitat Autonoma de Barcelona, 08193 Bellaterra, Barcelona, Spain.
}

faster than those of PMMA near the blend $T_{\mathrm{g}}$. The extreme difference in mobility between PEO and PMMA is a feature unique to this blend system. The dynamics of polyisoprene [PI] and poly(vinyl ethylene) [PVE] are separated by $2-5$ orders of magnitude, ${ }^{13,14}$ polystyrene [PS] and poly(vinyl methyl ether) [PVME] by 3-4 orders of magnitude, ${ }^{15}$ and polystyrene and poly(2,6-dimethyl phenylene oxide ${ }^{16,17}$ by next to nothing. Another unique feature of the PEO/PMMA blend system is the nearly composition independent dynamics displayed by PEO. ${ }^{9}$ In other miscible blends such as PI/PVE and PS/PVME, the segmental dynamics of both components change significantly with blend composition.

Several theoretical approaches have been proposed to explain distinct component dynamics in polymer blends. One theory ${ }^{18-21}$ suggests that thermal concentration fluctuations, which are most prominent in systems with a large $T_{\mathrm{g}}$ contrast and a small $\chi$, lead to a distribution of local compositions on length scales of around $10 \mathrm{~nm}$ near the glass transition temperature. Although no quantitative comparison between this model and experimental data on PEO/PMMA dynamics exists, we presented data for a $20 \mathrm{wt} \%$ blend in a previous publication ${ }^{22}$ supporting that concentration fluctuations are important in the dynamics of PEO in this system. The distribution of PEO relaxation times is extremely wide, and its behavior with spatial scale and temperature suggests this is linked to a wide distribution of local concentrations. The data of Lutz and co-workers, ${ }^{9}$ obtained over a range of compositions, does not support concentration 
fluctuations as an explanation of the fast and composition independent dynamics displayed by PEO in the blend. The second idea, proposed by Lodge and McLeish, ${ }^{23}$ asserts that distinct component dynamics result from an enhanced selfconcentration caused by chain connectivity: the local environment of an A segment is richer in itself because it is connected to two other A segments. The proposed relevant length scale for this self-concentration is the Kuhn length. This model has recently been tested on a large number of miscible blends where data is available and in many cases is successful. For PEO/ PMMA blends, the model fails to describe the dynamics. ${ }^{24}$ Since the fast and composition independent dynamics of PEO suggest that its mobility is decoupled from the PMMA matrix that surrounds it, Lutz and co-workers proposed that, due to the lack of side groups on PEO, it is able to relax without requiring PMMA rearrangement. Very recently, the same ${ }^{2} \mathrm{H}$ NMR data was analyzed in view of the coupling model of Roland and $\mathrm{Ngai}^{25}$ for blend dynamics. The basis for this model is the existence of a temperature insensitive crossover time $t_{\mathrm{c}}$ associated with the degree of cooperativity or coupling in a given system. The time $t_{\mathrm{c}}$ separates two relaxation regimes: a fast regime where intermolecular coupling is absent and a slow regime where cooperativity is important. This model, although not predictive, is able to describe the experimentally observed invariance of relaxation times for PEO with composition. This is attributed to relaxation times becoming comparable to the value of $t_{\mathrm{c}}$ (typically ${ }^{26-29} 2 \mathrm{ps}$ for pure polymers).

It is clear that some uncertainty remains regarding the nature of PEO dynamics when mixed with PMMA. In this contribution, we exploit the spatial sensitivity of quasi-elastic neutron scattering [QENS] to address the issue of the appropriate model for this purpose. We extend our prior measurements, which were for the 20\% PEO blend, to the range 10-30\% PEO and examine the ability of the chain connectivity and coupling models to describe the data as a function of spatial scale. We have considered the importance of concentration fluctuations for PEO in this blend in another publication. ${ }^{22}$ With respect to the coupling model, we are able to provide measured values of $t_{\mathrm{c}}$ and fast relaxation times specific to each blend. Both models fit the data only within limited spatial regions: $8-18 \AA$ for the chain connectivity model and 8-12 $\AA$ for the coupling model.

We highlight the motion of PEO using deuterium substitution. The large incoherent cross section of hydrogen compared to deuterium allows us to "hide" the motion of one component by substituting its hydrogen atoms for deuterium. In the present case, we measure blends of hydrogenated PEO [hPEO] and deuterated PMMA $\left[d_{8} \mathrm{PMMA}\right]$ to isolate PEO mobility. This differs from NMR measurements which require blends of deuterated PEO and hydrogenated PMMA. ${ }^{9}$ Because the $\chi$ parameter is small, there may be differences in phase behavior between these two mixtures, which will impact dynamics if concentration fluctuations are important for this system.

\section{Experimental Details}

Blend Preparation and Characterization. Hydrogenated PEO $\left[M_{\mathrm{w}}=463 \mathrm{~kg} / \mathrm{mol}\right]$ and perdeuterated PMMA $\left[M_{\mathrm{w}}=315 \mathrm{~kg} / \mathrm{mol}\right.$, $80 \%$ syndiotactic] with narrow molecular weight distributions were purchased from Polymer Standards Service. ${ }^{31}$ We prepared blends of 10,20 , and $30 \mathrm{wt} \%$ hPEO in $d_{8}$ PMMA by dissolving the two polymers in chloroform and casting them from solution. To ensure complete removal of the solvent, the samples were vaccuum-dried for a week at $350 \mathrm{~K}$. Differential scanning calorimetry [DSC] thermograms performed at a rate of $10 \mathrm{~K} / \mathrm{min}$, using a TA Instruments Q1000 differential scanning calorimeter, identified a single broad glass transition. This confirms that the blends are
Table 1. Glass Transition Temperatures Measured from DSC ${ }^{a}$

\begin{tabular}{cc}
\hline sample & $T_{\mathrm{g}}(\mathrm{K})$ \\
\hline $100 d_{8}$ PMMA & 391 \\
$10 \mathrm{hPEO} / 90 d_{8}$ PMMA & 365 \\
$20 \mathrm{hPEO} / 80 d_{8}$ PMMA & 344 \\
$30 \mathrm{hPEO} / 70 d_{8}$ PMMA & 315 \\
$100 \mathrm{hPEO}$ & 214 \\
${ }^{a}$ Compositions are given in wt $\%$. &
\end{tabular}

miscible and fully amorphous. The glass transition temperatures for the blends and the pure components are given in Table 1.

We obtained dynamic data using the disk chopper time-of-flight spectrometer $[\mathrm{DCS}]^{32}$ at the NIST Center for Neutron Research in Gaithersburg, MD. The spectrometer was operated at an incident wavelength of $4.2 \AA$ and at a resolution of $0.08 \mathrm{meV}$ (full width at half-maximum). Energy transfers in the range of -327 to +2.7 $\mathrm{meV}$ are detectable with this configuration. For this work, we concentrate on the quasi-elastic signal, that is, scattering events in the range -2 to $+2 \mathrm{meV}$. During the experiment, neutrons are bombarded in pulses onto the sample where they are scattered either elastically (their energy is unchanged) or inelastically (the neutron experiences an energy change). The flight times of scattered neutrons arriving at the detector array allow calculation of the energy transfer $(\hbar \omega)$. The scattering angle is also recorded to calculate the momentum transfer $(Q)$. In the configuration used, distances of $2-10 \AA$ and times of $1-40$ ps are investigated. The sample is annular in shape and held in a thin-walled aluminum can mounted onto a closed-cycle refrigerator. Sample thicknesses were kept around $0.2 \mathrm{~mm}$ to achieve transmissions of $\approx 90 \%$ and avoid multiple scattering effects. The instrumental resolution was measured using a vanadium sample at $295 \mathrm{~K}$ and the same instrument configuration. The measured QENS spectra collected over $6 \mathrm{~h}$ periods were corrected for detector efficiencies using software developed at NIST (Data Analysis and Visualization Environment, DAVE ${ }^{33}$ ). Subsequently, the scattering from the empty aluminum can and from the background were subtracted and the data were binned into $Q$-groups in the range $0.60-2.60 \AA^{-1}$.

\section{Results}

We investigate the mobility of PEO mixed with PMMA over a range of temperatures, 308-440 K, where PEO motion falls within the time scale of the DCS. Although QENS is normally not useful below the glass transition, in this case, the more mobile PEO remains in the window even below the blend $T_{\mathrm{g}}$, and thus, the chosen temperature range brackets the blend glass transitions. To assess changes in dynamics upon blending, we require data for pure PEO. Due to the large difference between the $T_{\mathrm{g}}$ and the melting temperature $\left(T_{\mathrm{m}}=334 \mathrm{~K}\right)$ of PEO, only one temperature was noncrystalline within the time window of DCS: $T=343 \mathrm{~K}$. At this temperature, we compare the dynamic structure factor, $\tilde{S}(Q, \omega)$, as a function of energy transfer $(E=$ $\hbar \omega)$, of all compositions, with that of pure PEO in Figure 1. The spectra are comprised of at least two line shapes: a tall Gaussian peak which corresponds to the instrumental resolution and defines the slowest motion that can be measured by the spectrometer, and additional broader peaks originating from proton motion. Since the line shapes of all the samples differ from that of vanadium, we infer that the mobility of PEO is within the time scale of DCS. Pure PEO is the most mobile of all compositions (has the broadest spectrum), confirming that the presence of PMMA retards PEO motion. To quantitatively assess differences in dynamics, it is advantageous to represent the data in the time domain, rather than in the energy (frequency) domain. In addition, the existence of more than one relaxation process cannot be directly discerned from the raw spectra, since they would all contribute to the total breadth of the peak. In the time domain, each of these processes will appear at different time scales. 

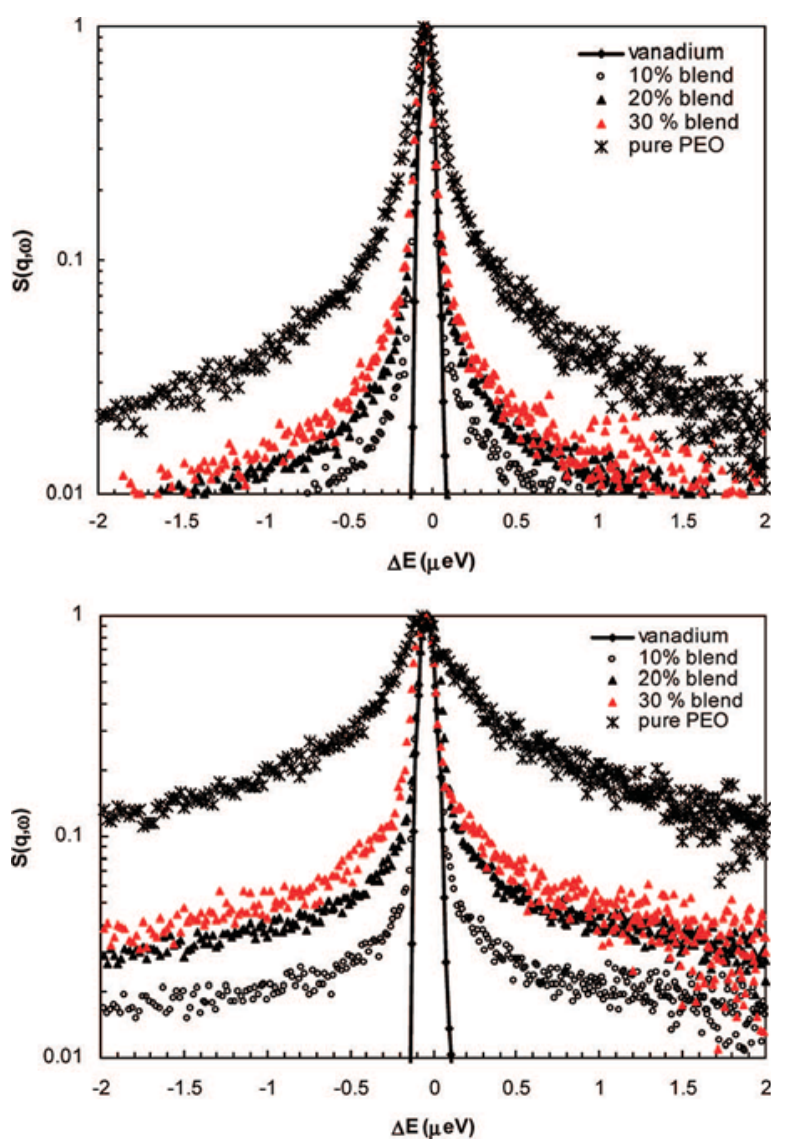

Figure 1. QENS spectra obtained with the DCS time-of-flight spectrometer at $Q=1.50 \AA^{-1}$ (top) and $Q=2.50 \AA^{-1}$ (bottom). All sample data are shown including the vanadium resolution. Scattering intensities have been normalized to the maximum intensity. Data correspond to temperatures of $343,345,348$, and $345 \mathrm{~K}$ for the 100 , 30,20 , and $10 \%$ hPEO samples, respectively. Only a section of the QENS spectra is shown, that is, energy transfers between -2 and +2 $\mathrm{meV}$.

We convert the raw data at each temperature and momentum transfer from the energy domain to the time domain by applying a discrete complex Fourier transform [FT] using the following expression:

$$
\tilde{S}(Q, t)=\sum_{k=1}^{N} \tilde{S}\left(Q, \omega_{k}\right) \exp \left(-i \omega_{k} t\right) \Delta \omega_{k}
$$

where $\omega_{k}$ is the angular frequency $\left(=E_{k} / \hbar\right)$ at each data point $k$ in a set with $N$ total data points. The measured self-intermediate scattering function $\tilde{S}(Q, t)$ is a convolution of the "true" selfintermediate scattering function $S(Q, t)$ and the resolution function $R(Q, t)$. This convolution in the frequency domain corresponds to a multiplication in the time domain:

$$
\tilde{S}(Q, t)=S(Q, t) \times R(Q, t)
$$

such that $S(Q, t)$ can be easily calculated. It is customary to express the self-intermediate function with values ranging from 0 to 1 ; thus, we also calculate $S(Q, 0)$ using eqs 1 and 2 and subsequently $S(Q, t) / S(Q, 0)=I(Q, t)$.

Figure 2 presents the same data as Figure 1, Fourier transformed into the time domain. The existence of two dynamic regimes is immediately apparent. The crossover between these two regimes is located at $1 \mathrm{ps}$ and is independent of temperature, composition, or spatial scale. From now on, we refer to the two relaxations in Figure 2 as the fast $\left(t<t_{\mathrm{c}}\right)$ and slow $\left(t>t_{\mathrm{c}}\right)$ processes and characterize them separately. The crossover time, which corresponds to $t_{\mathrm{c}}$ in Ngai's coupling model, is observed
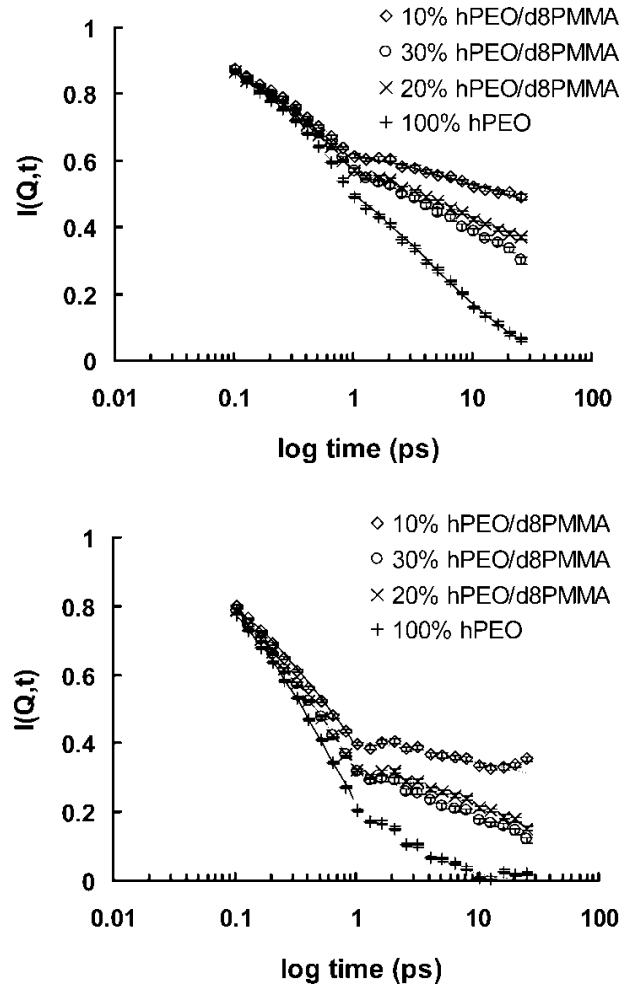

Figure 2. Self-intermediate scattering function for hPEO as a function of $d_{8}$ PMMA composition in the blend. Measurements are shown for $Q$ $=1.5 \AA^{-1}$ (top) and $Q=2.5 \AA^{-1}$ (bottom). Data correspond to temperatures of $343,345,348$, and $345 \mathrm{~K}$ for the $100,30,20$, and $10 \%$ hPEO samples, respectively. The lines represent fits with the KWW equation.

in pure PEO, and it has also been seen in other homopolymer melts. ${ }^{26-29}$ For this specific system, QENS measurements in this time range have not previously been made; however, molecular dynamics simulations did not show this crossover. ${ }^{12}$ We observe that the fast process is independent of the environment, whereas the slow process is not. This is the first measurement of the fast process in a polymer blend, and its invariance with composition is consistent with an intramolecular origin.

For the slow process, the data for the 20 and $30 \mathrm{wt} \%$ blends suggest a weak composition dependence, which is in agreement with the findings of Lutz and co-workers. ${ }^{9}$ This is not the case for the $10 \mathrm{wt} \%$ blend where PEO moves much slower than would be expected if the same composition dependence was continued. This could be due to labeling issues or an increasing contribution from the collective dynamics of deuterated PMMA. As mentioned above, we measure the hPEO/dPMMA blend whereas Lutz et al. measured the $d$ PEO/hPMMA blend. It is possible that the different composition dependence is a real feature that distinguishes the behavior of these two systems. We also consider the contribution from the coherent scattering of $d_{8}$ PMMA. Table 2 shows the estimated incoherent and coherent contributions to the total scattering (calculated from the scattering cross sections) from each polymer in the systems we measured. In the 20 and $30 \%$ samples, the incoherent scattering of hPEO clearly dominates, whereas in the $10 \%$ blend the coherent contribution from deuterium almost equals the incoherent scattering of hPEO. We anticipate that the collective dynamics of PMMA represented by this contribution will be too slow to be captured by DCS. In this case, the decay will not reflect the motion of PMMA, ${ }^{39}$ although it could contribute an elastic fraction.

To address this issue, we consider the time scale of collective motion of PMMA in the $10 \% \mathrm{hPEO} / d_{8} \mathrm{PMMA}$ blend. A direct measurement is impossible due to the presence of hPEO. We 
Table 2. Contributions to the Incoherent and Coherent Scattering from hPEO and $d_{8}$ PMMA in Each of the Samples Studied ${ }^{a}$

\begin{tabular}{|c|c|c|c|c|}
\hline sample & $\begin{array}{l}\text { incoherent scattering } \\
\text { from hPEO }(\%)\end{array}$ & $\begin{array}{l}\text { coherent scattering } \\
\text { from hPEO }(\%)\end{array}$ & $\begin{array}{l}\text { incoherent scattering } \\
\text { from } d_{8} \mathrm{PMMA}(\%)\end{array}$ & $\begin{array}{l}\text { coherent scattering } \\
\text { from } d_{8} \text { PMMA }(\%)\end{array}$ \\
\hline $10 \mathrm{hPEO} / 90 d_{8} \mathrm{PMMA}$ & 45.9 & 3.2 & 8.8 & 42.1 \\
\hline $20 \mathrm{hPEO} / 80 d_{8} \mathrm{PMMA}$ & 64.0 & 4.4 & 5.3 & 26.3 \\
\hline $30 \mathrm{hPEO} / 70 d_{8} \mathrm{PMMA}$ & 73.7 & 5.1 & 3.7 & 17.5 \\
\hline $100 \mathrm{hPEO}$ & 93.4 & 6.4 & 0.0 & 0.0 \\
\hline
\end{tabular}
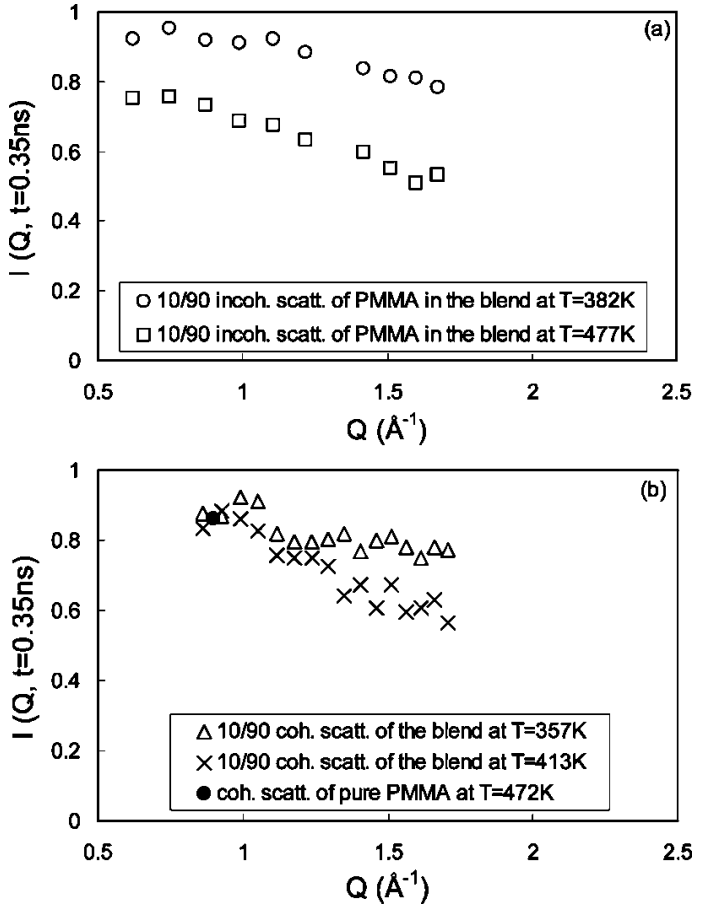

Figure 3. Values of the intermediate scattering function at $t \approx 350 \mathrm{ps}$ as a function of spatial scale: (a) incoherent scattering function of hPMMA $/ d_{4}$ PEO measured with the HFBS backscattering spectrometer ${ }^{30}$ and (b) coherent scattering function of pure $d_{8}$ PMMA and of a blend of $d_{8} \mathrm{PMMA} / d_{4} \mathrm{PEO}$.

have available three alternative estimates: pure $d_{8}$ PMMA, the $d_{4} \mathrm{PEO} / d_{8} \mathrm{PMMA}$ blend, and the hPMMA $/ d_{4} \mathrm{PEO}$ blend. Pure $d_{8}$ PMMA does not include the effect of blending, and thus, it will be too slow. In the $d_{4} \mathrm{PEO} / d_{8} \mathrm{PMMA}$ blend, coherent scattering from $\mathrm{PEO}$ will contribute such that this estimate will be too fast. In the hPMMA $/ d_{4} \mathrm{PEO}$ blend, the estimate will again be too fast due to the rotation of methyl groups, which are not present in collective motion, and the fact that collective motion is faster than self-motion. ${ }^{43}$

The indicators described above include data from two additional spectrometers. The hPMMA $/ d_{4} \mathrm{PEO}$ blend was measured ${ }^{30}$ using a backscattering spectrometer, and the $d_{8} \mathrm{PMMA}^{41,42}$ and $d_{4} \mathrm{PEO} /$ $d_{8} \mathrm{PMMA}^{41}$ samples were measured on two different neutron spin-echo spectrometers. To determine whether the mobility of PMMA in hPEO/ $d_{8}$ PMMA falls within the time window of DCS, in Figure 3, we compare the normalized scattering intensity $I(Q, t)$ for these three indicators at $t=350 \mathrm{ps}$, a time beyond the time window of DCS, which extends to $40 \mathrm{ps}$. This is a more stringent test than required, both because the time is beyond the time window of DCS and because hPMMA/d $d_{4} \mathrm{PEO}$ and $d_{4} \mathrm{PEO} / d_{8} \mathrm{PMMA}$ will be faster than the motion of interest. Data at two temperatures are presented: from the hPMMA/ $d_{4} \mathrm{PEO}$ sample in Figure $3 \mathrm{a}$ and from the $d_{8} \mathrm{PMMA}$ and $d_{4} \mathrm{PEO} /$ $d_{8}$ PMMA samples in Figure $3 \mathrm{~b}$. At low temperature and large spatial scales, only a small portion of the decay (less than $20 \%$ ) occurs before $350 \mathrm{ps}$, as indicated by $I(Q, t=0.35 \mathrm{~ns})$ values of 0.8 or higher. As a result, we conclude that the collective dynamics of PMMA would only contribute in the DCS time

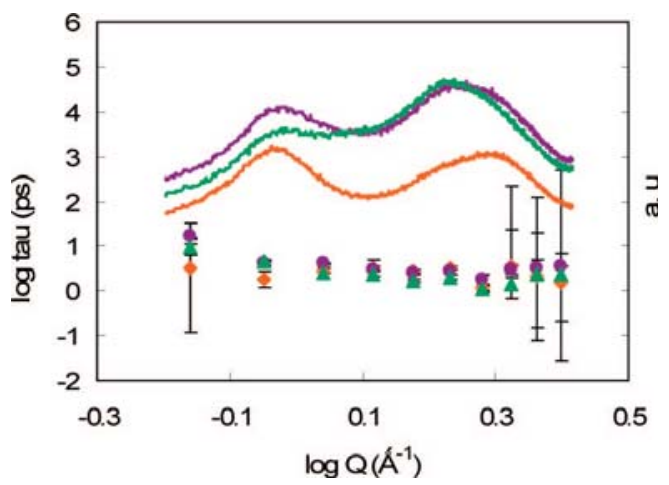

Figure 4. Spatial scale dependence of the characteristic relaxation times (obtained from KWW fits) of the slow process of PEO in all blends: (orange, $10 \%$, (purple, - 20\%, and (green, $\mathbf{\Delta}$ ) $30 \%$ blend. The lines show the static structure factor of the 10,20 , and $30 \%$ blends (same color code) ${ }^{41}$

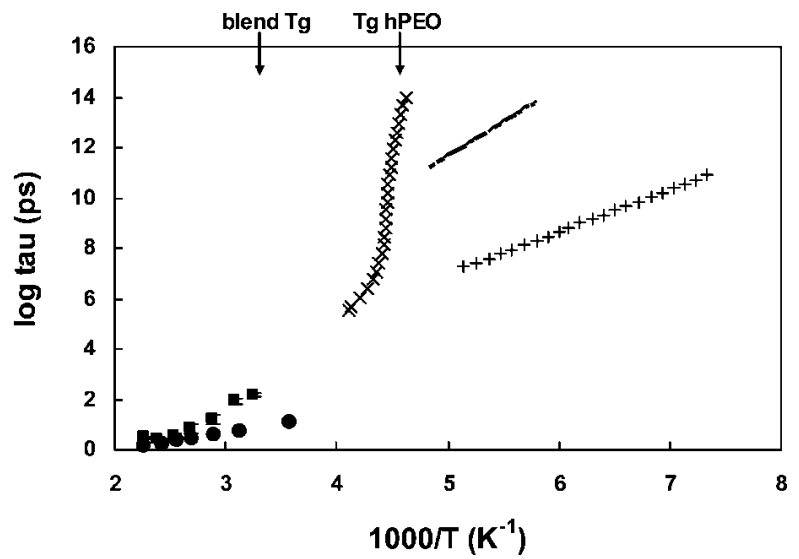

Figure 5. Temperature dependence of relaxation times obtained from KWW fits. Symbols correspond to (ם) QENS slow process data for the $20 \%$ blend at $Q=1.3 \AA^{-1}$ (this work), () QENS fast process data for the $20 \%$ blend at $Q=1.3 \AA^{-1}$ (this work), $(\times)$ dielectric spectroscopy data for the $\alpha$-relaxation of neat PEO, ${ }^{11}(+)$ dielectric spectroscopy data for the $\beta$-relaxation of PEO in a $20 \%$ blend, and $(-)$ dielectric spectroscopy data for the $\gamma$-relaxation of PEO in a $20 \%$ blend. ${ }^{11}$

window for data at high temperature and high $Q$. Specifically, if $T>425 \mathrm{~K}$ and $Q>1.5 \AA^{-1}$, we estimate that the PMMA component decays $20 \%$ or more by $350 \mathrm{ps}$, and as a result caution should be used in interpreting the data in this range. This applies to very few of our data sets, which span the temperature range $308-440 \mathrm{~K}$ and the spatial range $0.6-2.6$ $\AA^{-1}$. We also note that atomistic simulations of the coherent scattering signal of dPMMA in a blend with $25 \mathrm{wt} \% \mathrm{hPEO}^{12}$ at $400 \mathrm{~K}$ support this conclusion. For example, at $Q=1 \AA^{-1}$, the decay is around $15 \%$.

As a further test, we look for a variation of relaxation times in phase with the static structure factor $[S(Q)]$, which would be an indicator that collective motion is prominent. ${ }^{44}$ Figure 4 shows the spatial scale dependence of the slow relaxation times of all three blend compositions at $T=440 \mathrm{~K}$, where the largest contribution from coherent scattering of PMMA is expected. These times have been extracted using the Kolraush-Williams- 
Watts $[\mathrm{KWW}]^{34}$ expression, without accounting for an elastic fraction, as described below and denoted by eq 3. Also shown in the figure are the static structure factors of each blend. ${ }^{41}$ The relaxation times show no sign of a modulation with $Q$, but rather the $-2 / \beta$ scaling with a spatial scale characteristic of incoherent relaxation times ${ }^{44}$ is observed. The characteristic times obtained without accounting for an elastic fraction are similar for the $20 \%$ and $30 \%$ blends, which is consistent with prior measurements on this system. Characteristic times for the $10 \%$ blend are significantly slower, indicating that the larger contribution from PMMA is significant in this case. We conclude that the motion of PMMA does not contribute within the time scale of DCS, but that the elastic fraction must be taken into account when fitting data from the slow process.

\section{Characterization of the Fast and Slow Processes}

In this section we examine the two dynamic regimes observed for PEO in more detail. Specifically, we compare our QENS results to data from other experimental techniques and consider temperature and spatial dependencies of the parameters that characterize mobility. To obtain these parameters, we treat the fast and slow portions of the decay as two separate processes. Parameters for each are obtained using a KWW expression which describes translational motion in liquids. This expression, although empirical, describes the dynamics of neat polymers and blends ${ }^{14,15,22,35-38}$ and has been widely used to characterize mobility. In this description, the self-intermediate scattering function,

$$
I(Q, t)=A(Q, T) \exp \left[-\left(\frac{t}{\tau_{K W W}(Q, T)}\right)^{\beta(Q, T)}\right]
$$

is described by three parameters: the characteristic relaxation time $\tau_{K W W}$, the stretching exponent $\beta$ reflecting the distribution of relaxation times, and a prefactor $A$. The latter two can take values from 0 to 1 . All three parameters may be thermally and/ or spatially dependent. For the slow process, we take into account an elastic contribution from the immobile PMMA by introducing an additional fit parameter:

$$
\begin{array}{r}
I(Q, t)=A(Q, T)\left\{(1-E(Q, T)) \exp \left[-\left(\frac{t}{\tau_{K W W}(Q, T)}\right)^{\beta(Q, T)}\right]+\right. \\
E(Q, T)\}
\end{array}
$$

$E(Q, T)$ represents the fraction of the scattering arising from immobile protons in the PMMA component and is fixed at the values given in Table 2. Representative fit lines for both processes are included in Figure 2, and they illustrate that the resulting fits adequately describe the data.

Because of the large number of fitting parameters that are strongly coupled, we wish to set an error bar that describes the range of parameters that can describe the data. The error bars in our figures represent the maximum and minimum values of the parameters presented that can adequately describe the data. Error bars in $I(Q, t)$ [roughly the size of data markers in Figure 2] are calculated by synthesizing 500 data sets based on the uncertainty of the raw data, $\tilde{S}(Q, \omega)$, and performing the FT on each data set. Error bars on the KWW parameters (Figures 4-8) are assigned by generating 500 data sets within the error bars of $I(Q, t)$ and fitting them to the KWW equation. The fitting was accomplished using a nonlinear optimization solver CONOPT, accessed via General Algebraic Modeling System [GAMS]. The solver minimizes the squared difference between the predicted and measured values of $I(Q, t)$ by searching for the optimal values of $A, \beta$, and $\tau_{\mathrm{Kww}}{ }^{10}$ These values are obtained using a large number of initial guesses to find as many of the local minima (and therefore combinations of fit parameters) as possible. The significance of the error bars in Figure
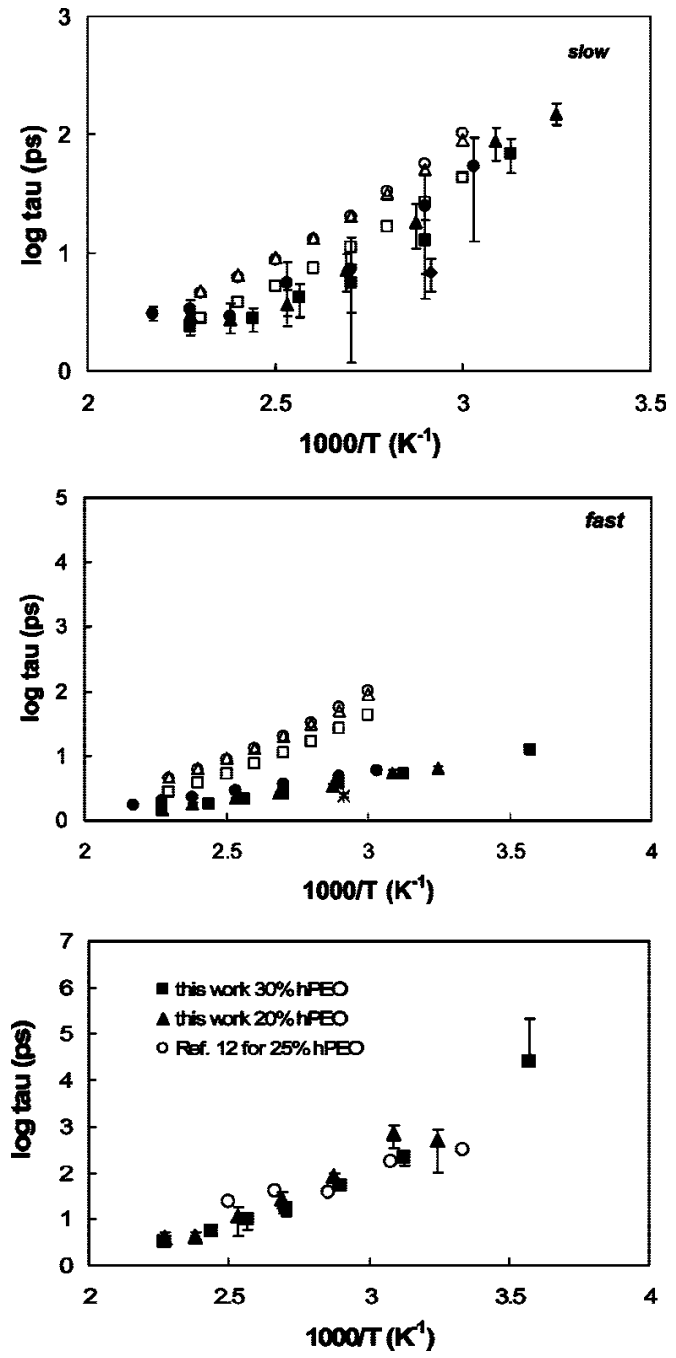

Figure 6. Composition dependence of the relaxation times obtained from KWW fits for the slow and fast processes of PEO in blends with PMMA. The figure compares data from QENS to data from NMR. ${ }^{9}$ Solid symbols correspond to QENS relaxation times obtained in this

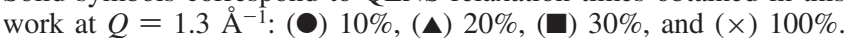
Empty symbols correspond to NMR segmental relaxation times: $(O)$ $10 \%,(\triangle) 20 \%$, and $(\square) 30 \%$. The bottom graph compares data (slow process) from QENS from this work at $Q=1.02 \AA^{-1}$ for (A) $20 \%$ and (ם) $30 \%$ blends, with data from a backscattering spectrometer in ref 12 for the (O) $25 \%$ blend.

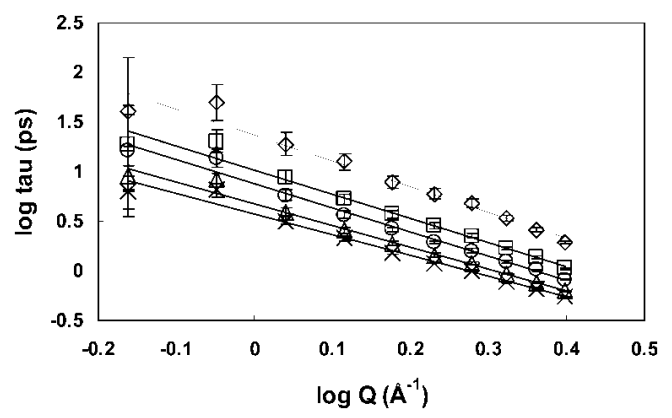

Figure 7. Spatial dependence of the relaxation times obtained from the KWW fits for the fast process for the $30 \%$ blend. Symbols correspond to different temperatures: $(\diamond) 280 \mathrm{~K}$, ( $\square) 320 \mathrm{~K}$, (○) 345 $\mathrm{K},(\triangle) 370 \mathrm{~K}$, and $(\times) 440 \mathrm{~K}$. The lines represent best fits through the data and have slopes ranging from -2.1 to -2.5 .

8 is that values outside the range illustrated cannot describe the data, even if the other two parameters are allowed to vary. An inability to accurately assign fit parameters due to limited decay is thus revealed as an excessively large error bar, which 

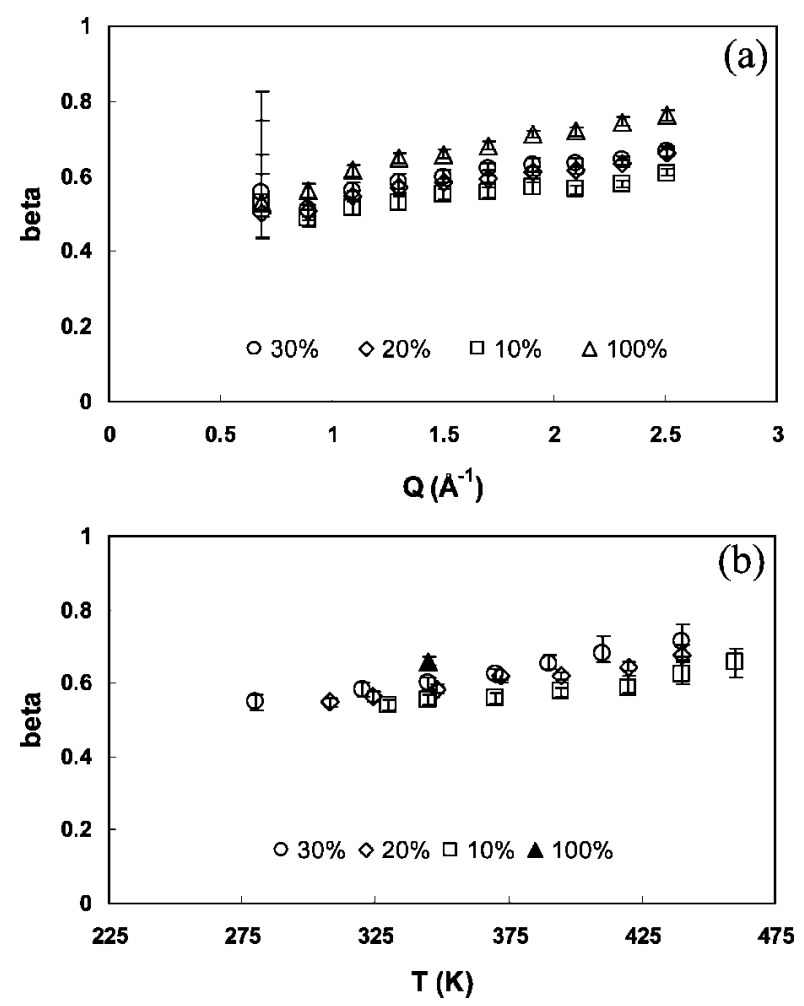

Figure 8. (a) Spatial and composition dependence of the stretching exponent for the fast process. The data correspond to $T=345 \mathrm{~K}$. (b) Temperature dependence of the stretching exponent for the fast process for all compositions. The data correspond to $Q=1.5 \AA^{-1}$.

indicates an unacceptable range between the minimum and maximum values possible for that parameter.

As discussed above, our results indicate the presence of two PEO relaxation processes in all blends, as well as in the pure melt, with a temperature and momentum transfer independent crossover time of $1 \mathrm{ps}$. In pure polymers, the nature of the fast process has been described as a vibrational ${ }^{62}$ process and an intramolecular relaxation process. ${ }^{63-66}$ Characteristic times for this process are weakly dependent on temperature and follow an Arrhenius scaling. The slow process refers to the structural relaxation of the polymer which is usually associated with the $\alpha$-relaxation. At low temperatures, the $\alpha$-relaxation, which shows a Vogel-like temperature dependence, is distinct from the $\beta$-relaxation, which is Arrhenius in temperature with an activation energy significantly greater than that of the fast process. In dielectric measurements, the $\alpha$ - and $\beta$-relaxations merge at a temperature somewhat above $T_{\mathrm{g}}$, and neutron measurements are typically associated with this region.

In Figure 8, we examine the temperature, composition, and spatial dependence of the fast and slow relaxation times and stretching parameters. The prefactor $A$ is considered a fit parameter but is not allowed to increase with increasing $Q$, as that would be unphysical. The relaxation times presented in these figures are those obtained directly from the KWW fits, and they have not been combined with the stretching parameter to form a "characteristic time" as is sometimes reported. We first place the relaxation times into context with data from other experimental techniques by comparing relaxation times from QENS with those from dielectric spectroscopy [DS, Figure 5] ${ }^{11}$ and $\mathrm{NMR}^{9}$ [Figure 6] as a function of temperature. Since QENS is the only technique that has spatial sensitivity, it is unclear which $Q$ value should be used for this purpose. We choose $Q=1.3$ $\AA^{-1}$ because previous comparisons ${ }^{46}$ between these techniques and neutron scattering have found agreement at spatial scales around $1 \AA^{-1}$, and our data most closely match NMR times in this range. A comparison between QENS and DS is shown in Figure 5 for the 20 wt \% PEO/PMMA blend. Dielectric spectroscopy captures two relaxation processes of PEO: the $\beta$-relaxation and another secondary process termed the $\gamma$-relaxation. The dielectric measurement is not able to discern the $\alpha$-relaxation of PEO when mixed with PMMA because, at the frequencies where it would be observed, the dielectric signal is dominated by the relaxation processes of PMMA. Instead, we provide relaxation times from the $\alpha$-relaxation of pure PEO. A comparison between DS and QENS data supports that the slow process is related to the $\alpha \beta$-relaxation of PEO, and the fast process is not observed in DS measurements. At the spatial scale shown, the activation energy obtained from QENS is $52.5 \mathrm{~kJ} /$ mol, whereas the activation energy of the $\beta$-relaxation from DS is $35.7 \mathrm{~kJ} / \mathrm{mol}$. Such change in the slope between the $\beta$ and merged $\alpha / \beta$ relaxations is consistent with observations of the $\alpha \beta$-relaxation for pure $\mathrm{PMMA}^{47}$ and for PMMA in a $20 \mathrm{wt} \%$ blend with PEO. ${ }^{10}$

We compare QENS and NMR relaxation times for both processes in Figure 6. For the slow process, reasonable agreement is observed for all three blends. The QENS data, although sharing the insensitivity to composition seen in NMR, is somewhat faster. In addition, NMR data show that the slow process is quite stretched, with $\beta$ values of 0.28 . ${ }^{9}$ At the spatial scale provided in the figure, $\beta$ values from QENS range between 0.35 and 0.40 at low temperature and increase to between 0.5 and 0.6 at the highest temperatures measured. Also shown in Figure 6 are times from a recent QENS study on PEO dynamics in this system. Those data were obtained from a backscattering spectrometer, ${ }^{12}$ but both characteristic times and stretching parameters are consistent with the present data, indicating that the two probe different time ranges of the same motion. Finally, we note that as the spatial scale increases, stretching parameters become smaller, in some cases as low as 0.20 . This is consistent with a wide distribution of characteristic times, which could arise from confinement effects. Such effects were observed in ref 12 at spatial scales larger than those measured by DCS.

In contrast to the slow process, characteristic relaxation times for the fast process are clearly smaller than those from NMR data. Although this "fast" process has previously been reported for pure polymers, the effect of mixing has not previously been examined. As suggested from the sample decays in Figure 2, relaxation times for the fast process are insensitive to environment. The temperature dependence of this process is Arrhenius with a small activation energy: $10-15 \mathrm{~kJ} / \mathrm{mol}$, independent of composition and $Q$. These values are consistent with conformational transitions in pure polymers [PI, PB, PVC]. For pure polymers, the fast process is usually associated with unhindered motion within a local cage, the extent of which is described by the Debye-Waller factor. The decay function associated with local cage vibrations is exponential (i.e., $\beta=1$ ), with characteristic times that follow a $Q^{2}$ scaling. ${ }^{49}$ Figure 7 shows the spatial dependence of fast relaxation times for the $30 \%$ blend at various temperatures. In agreement with local cage exploration, the exponent in this scaling varies between 2.0 and 2.5. The decay is not a simple exponential: values of the stretching exponent fall in the range $0.5-0.8$ for pure PEO and PEO mixed with PMMA at all temperatures. This is illustrated in Figure 8 where we present fast process stretching exponents as a function of spatial scale [Figure 8a] and temperature [Figure 8b]. The fast process of pure PEO is also characterized by a stretched exponential decay, ruling out the possibility that blending is the cause of the stretched characteristic. The fast process in PEO is very prominent; that is, it decays significantly before the slower process intervenes. This additional decay may reveal nonexponential behavior not otherwise observable. One possible origin is a distribution of local cage sizes, or mean squared 

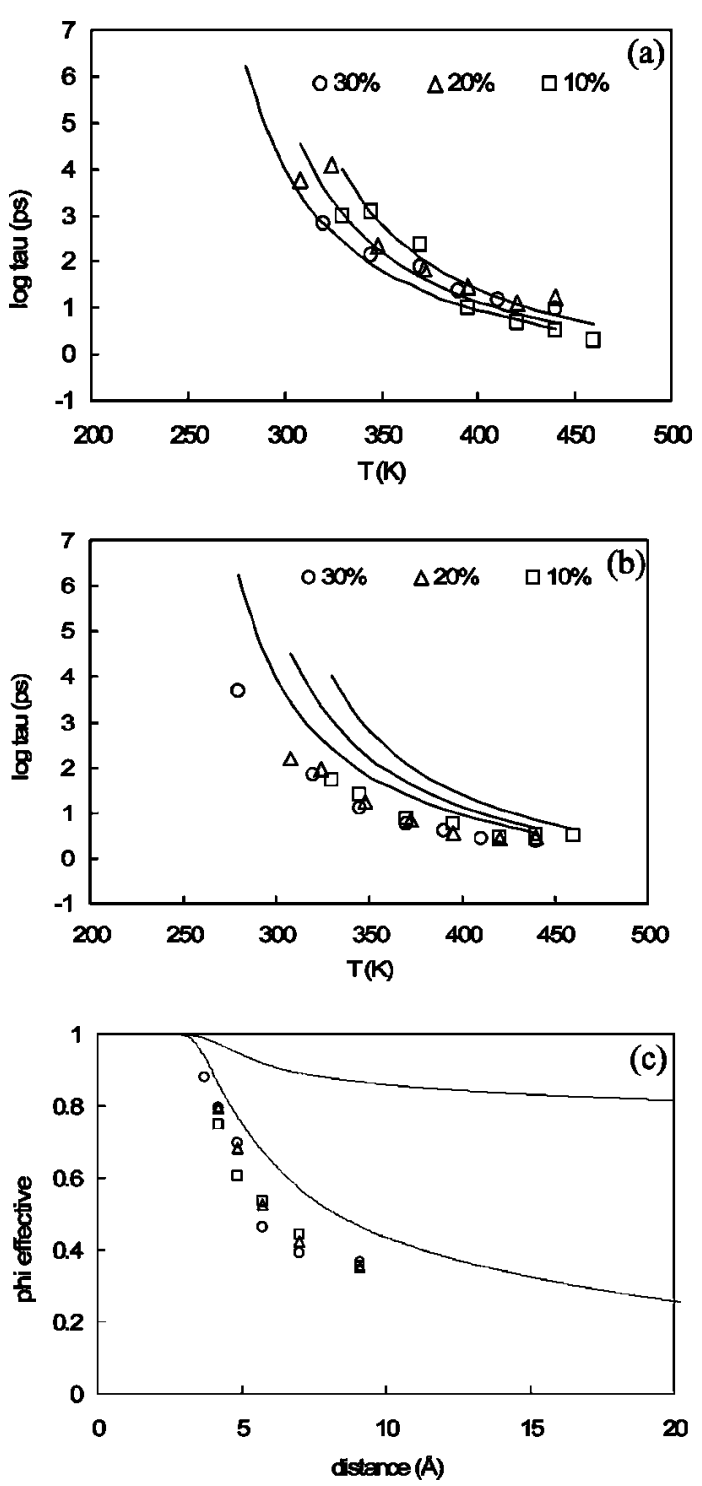

Figure 9. Comparison between the Lodge-McLeish model and QENS data for PEO in PMMA blends fit with the predicted value: $\phi_{\text {self }}=$ 0.22 at (a) $Q=0.69 \AA^{-1}$ and (b) $Q=1.3 \AA^{-1}$. Symbols represent the QENS data, and lines represent the LM model predictions. Panel (c) shows the spatial dependence of $\phi_{\text {eff }}$ obtained by fitting the LM model to QENS data at three different $Q$ values: $(O) 30 \%$ blend, $(\triangle) 20 \%$ blend and, ( $\square$ ) $10 \%$ blend; the lines are values calculated from molecular dynamics simulation ${ }^{53}$ for PEO (solid) and PMMA (dashed) in a $20 \%$ PEO blend.

displacements, which when averaged appears as a nonexponential decay.

\section{Comparison to Dynamic Models}

As discussed in the Introduction, three models have been proposed to explain component dynamics in polymer mixtures. In this work, we consider two of these, the chain connectivity model and the coupling model, and use the spatial sensitivity of QENS to assess their applicability at various length scales. The controlling length scale in the dynamics of mixtures is an open question. The widespread success of the chain connectivity model, with respect to both the number of systems for which it provides an adequate description of obtained data and the range of data it can correlate, suggests that a length scale of dimensions comparable to the Kuhn length controls dynamic response in mixtures regardless of the spatial extent of the technique used to measure this response. A technique such as QENS should thus reveal that the self-concentration is invariant as the size scale of the observed region changes. We expect different behavior in the case of PEO/PMMA, because the chain connectivity model is not able to describe available data. This behavior may provide some insight as to why chain connectivity does not work in this system. The coupling model does not rely on a specific length scale, but the primitive relaxation which couples to the segmental relaxation should be prominent on small spatial scales. It may thus be expected that this model will describe the data at spatial scales large enough to include significant environmental contacts.

The Chain Connectivity Model. In the chain connectivity model, ${ }^{23}$ segmental relaxation is controlled by the composition of a local region with dimensions comparable to the Kuhn length, $l_{\mathrm{K}}$, termed the effective concentration. The selfconcentration of a polymer segment centered in this local region is dictated by chemical bonding and chain flexibility. This selfconcentration, $\phi_{\text {self }}$, causes the effective concentration

$$
\phi_{\text {eff }}^{i}=\phi_{\text {self }}^{i}+\left(1-\phi_{\text {self }}^{i}\right) \phi_{\text {bulk }}
$$

to vary from the bulk composition $\left(\phi_{\text {bulk }}\right)$. The self-concentration is calculated from the volume fraction occupied by a Kuhn length $\left(l_{\mathrm{K}}\right)$ of repeat units inside a volume of dimension comparable to the Kuhn length. A cubic volume is normally used for this purpose; in our case, a spherical volume $\left[\pi l_{\mathrm{K}}{ }^{3} / 6\right]$ best describes QENS measurements, such that

$$
\phi_{\mathrm{self}}^{i}=\frac{6 C_{\infty} M_{0}}{k \rho N_{\mathrm{AV}} \pi l_{\mathrm{K}}^{3}}
$$

The required parameters characteristic ratio $C_{\infty}$, molecular weight of one repeat unit $M_{0}$, number of backbone bonds in a repeat unit $k$, bulk density $\rho$, Avogadro's constant $N_{\mathrm{AV}}$, and Kuhn length $l_{\mathrm{K}}$ are all numerical constants or pure component parameters, making this theory predictive in the sense that blend properties may be estimated based on pure component inputs. The dynamics of each component depend on the effective concentration through an effective glass transition temperature, normally determined using the Fox equation evaluated at $\phi_{\text {eff: }}$ :

$$
\frac{1}{T^{i}\left(\phi_{\text {eff }}\right)}=\frac{\phi_{\text {eff }}^{i}}{T_{\mathrm{g}}^{\mathrm{A}}}+\frac{1-\phi_{\mathrm{eff}}^{i}}{T_{\mathrm{g}}^{\mathrm{B}}}
$$

Because the effective concentrations of the two components differ, the effective glass transition temperatures also differ, which causes a separation of segmental relaxation times. These times are calculated using the Vogel-Tammann-Fulcher (VTF) framework, where pure component values of the constants $\tau_{\infty, i}$ and $B_{i}$ are used. The influence of the effective concentration and thus the second component enters through the third constant: the Vogel temperature. In the relationships used:

$$
\begin{gathered}
\tau_{\text {seg }, i}(\phi, T)=\tau_{\infty, i} \exp \left[\frac{B_{i}}{T-T_{0, i}(\phi)}\right] \\
T_{0, i}(\phi)=T_{0, i}+T_{g}^{i}\left(\phi_{\text {eff }}^{i}\right)-T_{g, i}^{\text {pure }}
\end{gathered}
$$

$i$ represents homopolymer $\mathrm{A}$ or $\mathrm{B}, \tau_{\infty, t}$ and $B_{i}$ are the VTF parameters that describe the temperature dependence of the segmental relaxation of homopolymer $i$, and $T_{0, i}$ and $T_{0, i}(\phi)$ are the Vogel temperatures for homopolymer $i$ and the blend, respectively.

The chain connectivity model is able to correlate slow QENS relaxation times over a range of spatial scales clustered around the Kuhn length of PEO, 7-18 $\AA$, but the predicted value of the self-concentration provides a reasonable description only at the large end of this spatial range. As an example, a comparison between the model prediction and our QENS data is shown in Figure 9a for all compositions, where the predicted 

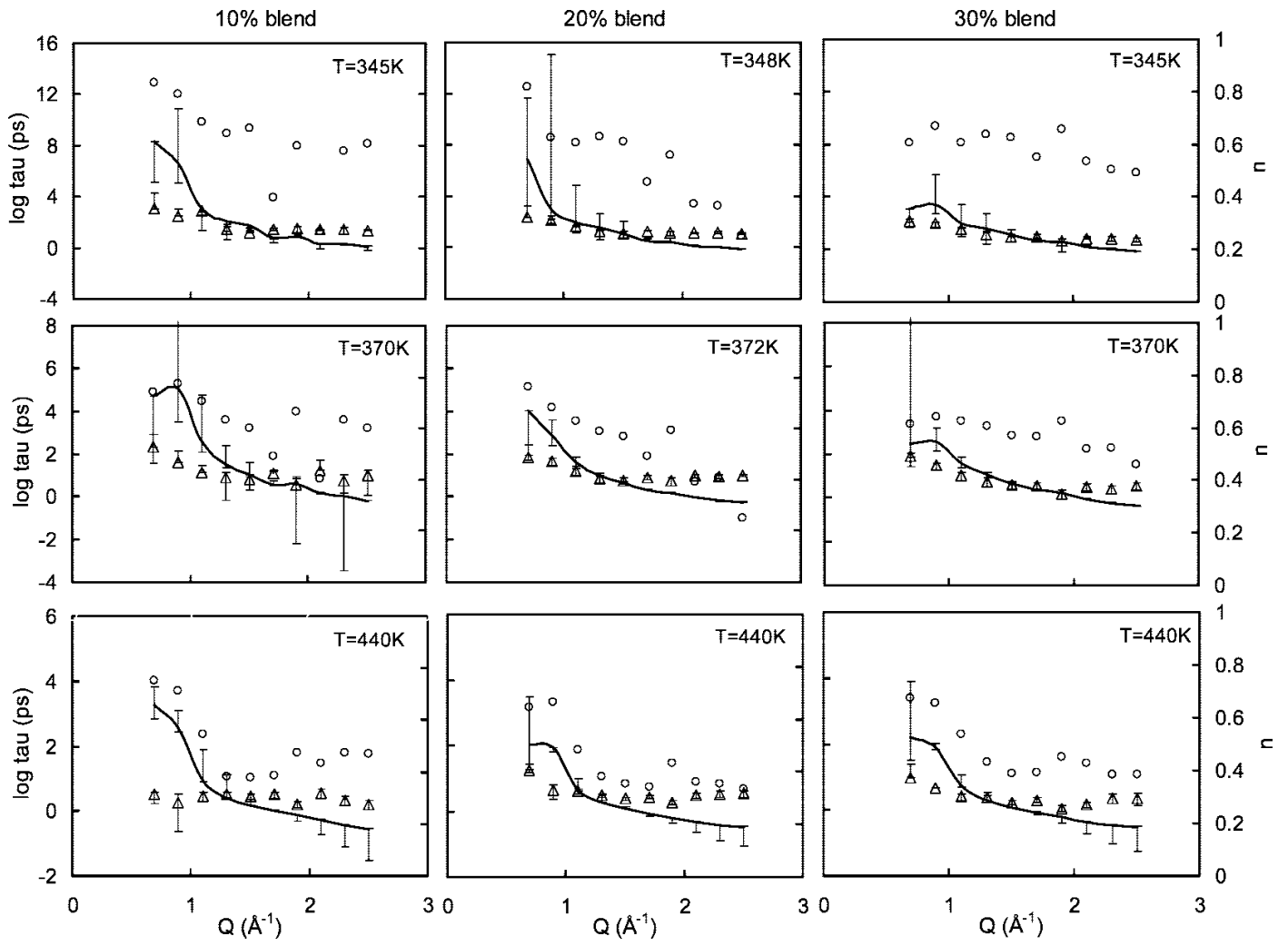

Figure 10. Comparison of relaxation times obtained from fits to the KWW equation from QENS ( $\checkmark$ with solid error bars) and those calculated using Ngai's coupling model (line with dashed error bars). Also shown are the values of the coupling parameter used $(O)$.

value, $\phi_{\text {self }}=0.22$, is used. The spatial scale illustrated, $Q=$ $0.69 \AA^{-1}$, is the spatial scale at which the best fit is obtained, and it describes a sphere with diameter $\approx 18 \AA$, somewhat larger than the Kuhn length of PEO. The data are best described for the $20 \%$ blend. For this comparison, we required VTF parameters for $\mathrm{PEO}^{9}\left[B=748.5 \mathrm{~K}, T_{0}=166 \mathrm{~K}\right.$, and $\left.\tau=0.095 \mathrm{ps}\right]$ and parameters for the calculation of $\phi_{\text {self }}\left[C_{\infty}=6.7, M_{0}=44\right.$ $\mathrm{g} / \mathrm{mol}, k=3, \rho=1.07 \mathrm{~g} / \mathrm{cm}^{3}, N_{\mathrm{AV}}=6.023 \times 10^{23} \mathrm{~mol}^{-1}$, and $\left.l_{\mathrm{K}}=1.1 \times 10^{-7} \mathrm{~cm}\right] .{ }^{51}$ As the spatial scale is decreased, $\phi_{\text {self }}$ $=0.22$ no longer describes the data, although it can be correlated by allowing $\phi_{\text {self }}$ to vary. This is illustrated in Figure $9 \mathrm{~b}$, again using the predicted value of $\phi_{\text {self }}=0.22$, where the illustrated spatial scale, $Q=1.3 \AA^{-1}$, describes a sphere approximately $10 \AA$ in diameter. As is apparent from the figure, the composition dependence of the data is less than that of the Lodge-McLeish $[\mathrm{LM}]$ prediction, and agreement only becomes quantitative at high temperatures.

Because of the spatial resolution in QENS, we are able to evaluate the LM model and obtain an estimate of $\phi_{\text {eff }}$ as a function of length scale. To do so, we treat $\phi_{\text {self }}$ as a fit parameter, allowing it to vary with composition and spatial scale. With this approach, reasonable fits can be obtained in the $Q$-range between 0.69 and $1.5 \AA^{-1}$. This differs from the treatment leading to Figure $9 \mathrm{a}$ in which $\phi_{\text {self }}$ was constrained to the predicted value for all three blend compositions. The selfconcentrations required to describe the data were used with eq 4 to determine the effective concentration, and we present the results in Figure 9c. Since the predicted value of $\phi_{\text {self }}$ produces a good fit at larger spatial scales, with quantitative accuracy most apparent for the $20 \%$ blend, the connection between this graph and Figure 9a is the 20\% data at $r=9.1 \AA[Q=0.69$ $\AA^{-1}$ ] where the effective concentration corresponding to $\phi_{\text {self }}$ $=0.22$ is 0.38 . The other compositions take different values of $\phi_{\text {self }}$, interestingly, such that the effective concentrations of all three blends are coincident. The effective concentrations corresponding to $\phi_{\text {self }}=0.22$ for the other two blends are $\phi_{\text {eff }}=$
0.46 [for the $30 \%$ blend] and 0.30 [for the $10 \%$ blend], a difference not observed in Figure 9c. As the length scale of the measurement is decreased, the effective concentration does not remain constant, but rather it varies considerably within the spatial range where the model is able to fit the data. This does not support a constant length scale which controls the influence of mixing on dynamics, but it does vary as one would expect the local concentration to vary with spatial scale. At small distances, only self-contacts are possible and we expect $\phi_{\text {eff }}$ to be unity, dropping off as the first intermolecular contacts are encountered and eventually reaching the bulk blend composition. This is confirmed by MD simulations on this system, ${ }^{53}$ in which the effective concentration may be directly calculated as a function of local volume size, and these curves for both blend components are presented in the figure. Similar results were obtained from MD simulations of polyolefin blends. ${ }^{52}$

The Coupling Model. The coupling model developed by Roland and $\mathrm{Ngai}^{25}$ describes the segmental dynamics of polymer blends as a coupling of a primitive relaxation, that is, one without influence of local environment, with the new environment introduced by the second component. The model is based on their coupling model ${ }^{54-57}$ for neat polymers. The intermediate scattering function of a neat polymer follows a stretched exponential decay:

$$
\phi(t)=\phi_{0} \exp \left[-(t / \tau)^{1-n}\right]
$$

The coupling parameter $n$ describes the intermolecular coupling, and $\tau$ is the segmental relaxation time. The primitive relaxation is introduced using

$$
\tau(n)=\left(t_{\mathrm{c}}^{n} \tau_{0}\right)^{1 / 1-n}
$$

where $t_{\mathrm{c}}$ is the time scale at which coupling begins and $t_{0}$ is the primitive relaxation time. In the context of the QENS measurements presented in this work, $\tau(n)$ is the slow process relaxation time, $\tau_{0}$ is the fast process relaxation time, and $t_{\mathrm{c}}$ is the crossover 
time from fast to slow. QENS provides all three parameters directly, relieving the need to use pure component parameters or estimates and providing a more stringent test of the model. Our measurements reveal that $t_{\mathrm{c}}=1 \mathrm{ps}$ for all systems at all temperatures and spatial scales. Predictions of $\tau(n)$ are made with $t_{\mathrm{c}}=1 \mathrm{ps}$, with $\tau_{0}$ equal to the relaxation time of the fast process at a given temperature, momentum transfer, and blend composition and $n$ determined from the stretching exponent $\beta$ (obtained from fitting the intermediate scattering function to the KWW equation). Because the model requires blend data as input, it is not predictive.

We compare relaxation times calculated using the coupling model as described above with those from the slow QENS process as a function of spatial scale in Figure 10. Three temperatures and all compositions are considered. Also shown are the values of the coupling parameter used in the calculation. The error bars in the calculated relaxation times represent the spread based on the uncertainty in the values of $\beta$. The agreement between the model predictions and the data is reasonable. We note that, as with comparison to the chain connectivity model, the coupling model works best within a narrow region clustered around the Kuhn length of PEO. A previous comparison of the model to NMR data ${ }^{25}$ showed good agreement using a typical value of $t_{\mathrm{c}}$ for polymers and pure PEO relaxation times for $\tau_{0}$. We can now confirm that the value of $t_{\mathrm{c}}$ used in this comparison is accurate [ 2 ps vs $1 \mathrm{ps}$ ] and that using pure relaxation times for $\tau_{0}$ is reasonable because the fast process is insensitive to environment. The present comparison is more accurate, since it uses measured values for these parameters. Agreement between predicted and measured relaxation times is worst below the $T_{\mathrm{g}}$ of PMMA and at small spatial scales. We have previously reported unusual behavior in this regime, in that $\beta$ changes considerably and tends to pure PEO values. Agreement also deteriorates for spatial scales above $\sim 1$ $\AA^{-1}$. This corresponds to a sphere of diameter $\sim 12 \AA$, whereas the chain connectivity model remains accurate up to $18 \AA$. We note that it has been reported that QENS data for this system indicate confinement below the $T_{\mathrm{g}}$ of PMMA, where the system may be regarded as mobile PEO segments "confined" by the immobile PMMA. ${ }^{12}$ It may be that the character of the observed process changes under these conditions (PEO confined between immobile PMMA segments) and is no longer represented by models designed to describe the $\alpha$-relaxation. The $\alpha$-relaxation of PEO has been reported to disappear entirely when confined in other systems, which is consistent with all the above evidence. ${ }^{61}$

\section{Conclusions}

We have used neutron scattering to study the dynamics of PEO in blends with PMMA. We observe two distinct processes occurring at different time scales. Relaxation times for the slow process correspond to DS and NMR measurements of the segmental or $\alpha$-relaxation of PEO in the PEO/PMMA blend. Relaxation times for the fast process are much smaller than previous measurements of the PEO segmental relaxation and depend differently on temperature. This process, previously observed in pure polymers, is examined for the first time as a function of environment. We find it is insensitive to mixing, in keeping with its interpretation as exploration of a local cage.

We use the spatial selectivity of QENS to examine two models used for blend dynamics: the chain connectivity model and the coupling model. In both cases, we assess the ability of the models to describe the motion of PEO over spherical regions varying in diameter from 6 to $22 \AA$. We find that both models describe PEO motion in limited regions: $8-18 \AA$ for the chain connectivity model, and 8 to $12 \AA$ for the coupling model. The chain connectivity model yields effective compositions that vary with spatial scale, in contrast to the idea of a single length scale controlling the dynamics of mixtures.

Acknowledgment. Financial support for this work was provided by the National Science Foundation, Polymers Program, through a CAREER Grant DMR-0134910. This work used facilities supported in part by the National Science Foundation under Agreement No. DMR-0454672.

\section{References and Notes}

(1) Ito, H.; Russell, T. P.; Wignall, G. D. Macromolecules 1987, 20, 2213 2220.

(2) Hopkinson, I.; Kiff, F. T.; Richards, R. W.; King, S. M.; Farren, T. Polymer 1995, 36, 3523-3531.

(3) Colby, R. H. Polymer 1989, 30, 1275-1278.

(4) Zawada, J. A.; Ylitalo, C. M.; Fuller, G. G.; Colby, R. H.; Long, T. E. Macromolecules 1992, 25, 2896-2902.

(5) Straka, J.; Schmidt, P.; Dybal, J.; Schneider, V.; Spevácek, J. Polymer 1995, 36, 1147-1155.

(6) Lartigue, C.; Guillermo, A.; Cohen-Addad, J. P. J. Polym. Sci., Part B: Polym. Phys. 1997, 35, 1095-1105.

(7) Schantz, S. Macromolecules 1997, 30, 1419-1425.

(8) Dionisio, M.; Fernandes, A. C.; Mano, J. F.; Correia, N. T.; Sousa, R. C. Macromolecules 2000, 33, 1002-1011.

(9) Lutz, T. R.; He, Y.; Ediger, M. D.; Cao, H.; Lin, G.; Jones, A. A. Macromolecules 2003, 36, 1724-1730.

(10) García Sakai, V.; Chen, C.; Maranas, J. K.; Chowdhuri, Z. Macromolecules 2004, 37, 9975-9983.

(11) Jin, X.; Zhang, S.; Runt, J. Macromolecules 2004, 37, 8110-8115.

(12) Genix, A.-C.; Arbe, A.; Alvarez, F.; Colmenero, J.; Willner, L.; Richter, D. Phys. Rev. E 2005, 72, 031808.

(13) Chung, G. C.; Kornfield, J. A.; Smith, S. D. Macromolecules 1994, 27, 5729-5741.

(14) Min, B. C.; Qiu, X. H.; Ediger, M. D.; Pitsikalis, M.; Hadjicristidis, N. Macromolecules 2001, 34, 4466-4475.

(15) Cendoya, I.; Alegría, A.; Alberdi, J. M.; Colmenero, J.; Grimm, H.; Richter, D.; Frick, B. Macromolecules 1999, 32, 4065-4078.

(16) Chin, Y. H.; Zhang, C.; Wang, P.; Inglefield, P. T.; Jones, A. A.; Kambour, R. P.; Blender, J. T.; White, D. M. Macromolecules 1992, 25, 3031-3038.

(17) Chin, Y. H.; Jones, A. A.; Inglefield, P. T. Macromolecules 1993, 26, 5372-5378.

(18) Zetsche, A.; Fischer, E. W. Acta Polym. 1994, 45, 168-175.

(19) Katana, G.; Fischer, E. W.; Hack, Th.; Abetz, V.; Kremer, F. Macromolecules 1995, 28, 2714-2722.

(20) Kumar, S. K.; Colby, R. H.; Anastasiadis, H.; Fytas, G. J. Chem. Phys. 1996, 105, 3777-3788.

(21) Kamath, S.; Colby, R. H.; Kumar, S. K.; Karatasos, K.; Floudas, G.; Fytas, G.; Roovers, J. E. L. J. Chem. Phys. 1999, 111, 6121-6128.

(22) García Sakai, V.; Maranas, J. K.; Chowdhuri, Z.; Peral, I.; Copley, J. R. D. J. Polym. Sci., Part B: Polym. Phys. 2005, 43, 2914-2923.

(23) Lodge, T. P.; McLeish, T. C. B. Macromolecules 2000, 33, 52785284.

(24) He, Y.; Lutz, T. R.; Ediger, M. D. J. Chem. Phys. 2003, 119, 99569965.

(25) Ngai, K. L.; Roland, C. M. Macromolecules 2004, 37, 2817-2822.

(26) Zorn, R.; Arbe, A.; Colmenero, J.; Frick, B.; Richter, D.; Buchenau, U. Phys. Rev. E 1995, 52, 781-795.

(27) Colmenero, J.; Arbe, A.; Coddens, G.; Frick, B.; Mijangos, C.; Reinecke, H. Phys. Rev. Lett. 1997, 78, 1928-1931.

(28) Colmenero, J.; Arbe, A. Phys. Rev. E 1998, 57, 13508-13513.

(29) Kanaya, T.; Kawaguchi, T.; Kaji, K. Macromolecules 1999, 32, 1672 1678.

(30) Liu, J.; García Sakai, V.; Maranas, J. K.; Chowdhuri, Z. Macromolecules 2006, 39, 2866-2874.

(31) The use of commercial products identified in this paper does not imply recommendation or endorsement by the National Institute of Standards and Technology, nor does it imply that the materials or equipment identified are necessarily the best available for the purpose.

(32) Copley, J. R. D.; Cook, J. C. Chem. Phys. 2003, 292, 477-485.

(33) The IDL-based program can be found at http://www.ncnr.nist.gov/ dave.

(34) Williams, G.; Watts, D. C. Trans. Faraday Soc. 1970, 66, 80.

(35) Alegria, A.; Elizetxea, C.; Cendoya, I.; Colmenero, J. Macromolecules 1995, 28, 8819-8823.

(36) Arbe, A.; Alegria, A.; Colmenero, J.; Hoffmann, S.; Willner, L.; Richter, D. Macromolecules 1999, 32, 7572-7581.

(37) Doxastakis, M.; Kitsiou, M.; Fytas, G.; Theodorou, D. N.; Hadjicristidis, N.; Meier, G.; Frick, B. J. Chem. Phys. 2000, 112, 8687-8694.

(38) Hoffmann, S.; Willner, L.; Richter, D.; Arbe, A.; Colmenero, J.; Farago, B. Phys. Rev. Lett. 2000, 85, 772-775. 
(39) Talón, C.; Smith, G.; Price, D.; Copley, J. R. D. J. Phys. Chem. B 2004, 108, 5120-5126.

(40) de Gennes, P. G. Physica 1959, 25, 825-839.

(41) Farago, B.; Chen, C.; Maranas, J. K.; Kamath, S.; Colby, R. H.; Pasquale, A. J.; Long, T. E. Phys. Rev. E 2005, 72, 031809.

(42) García Sakai, V.; Chen, C.; Maranas, J. K.; Rosov, N. Unpublished data.

(43) Neelakantan, A.; Maranas, J. K. J. Chem. Phys. 2004, 120, 465-474.

(44) Farago, B.; Arbe, A.; Colmenero, J.; Faust, R.; Buchenau, U.; Richter, D. Phys. Rev. E 2002, 65, 051803.

(45) Neelakantan, A.; Maranas, J. K. J. Chem. Phys. 2004, 120, $1617-$ 1626.

(46) Colmenero, J.; Alegria, A.; Alberdi, J. M.; Alvarez, F.; Frick, B. Phys. Rev. B 1991, 44, 7321-7329.

(47) Bergman, R.; Alvarez, F.; Alegria, A.; Colmenero, J. J. Non-Cryst. Solids 1998, 235, 580-583.

(48) Graessley, W. W.; Krishnamoorti, R.; Balsara, N. P.; Fetters, L. J. Lohse, D. L.; Schultz, D. N.; Sissano, J. A. Macromolecules 1993, 26, 1137-1143.

(49) Higgins, J. S.; Benoit, H. C. Polymers and neutron scattering; Oxford University Press: Oxford, 1994.

(50) Kim, E.; Mattice, W. L. J. Chem. Phys. 1994, 101, 6242-6254.

(51) Rubinstein, M.; Colby, R. H. Polymer physics; Oxford University Press: New York, 2003.

(52) May, A.; Maranas, J. K. J. Chem. Phys. 2006, 125, 024906.
(53) Chen, C.; Maranas, J. K. In preparation.

(54) Roland, C. M.; Ngai, K. L. Macromolecules 1991, 24, 2261-2265.

(55) Roland, C. M.; Ngai, K. L. J. Rheol. 1992, 36, 1691-1706.

(56) Ngai, K. L.; Roland, C. M. Macromolecules 1992, 25, 363-367.

(57) Roland, C. M.; Ngai, K. L. Macromolecules 2001, 34, 6159-6160.

(58) Lodge, T. P.; Wood, E. R.; Haley, J. C. J. Polym. Sci., Part B: Polym. Phys. 2006, 44, 756-763.

(59) Bedrov, D.; Smith, G. D. Macromolecules 2005, 38, 10314-10319.

(60) Maranas, J. K. Curr. Opin. Colloid Interface Sci. 2007, 12, 29-42.

(61) Elmahdy, M. M.; Chrissopoulou, K.; Afratis, A.; Floudas, G.; Anastasiadis, S. H. Macromolecules 2006, 39, 5170-5173.

(62) Buchenau, U.; Monkenbusch, M.; Stamm, M.; Majkrzak, C. F.; Nücker, N. In Polymer Motion in Dense Systems; Richter, D., Springer, T., Eds.; Springer Proceedings in Physics 29; Springer: Berlin, 1988; p 138.

(63) Götze, W. In Liquids, Freezing and the Glass Transition; Hansen, J. P., Levesque, D., Zinn-Justin, J., Eds.; North-Holland: Amsterdam, 1991; p 287.

(64) Knaak, W.; Mezei, F.; Farago, B. Europhys. Lett. 1988, 7, 529-536.

(65) Frick, B.; Farago, B.; Richter, D. Phys. Rev. Lett. 1990, 64, 29212924.

(66) Wuttke, J.; Kiebel, M.; Bartsch, J.; Fujara, F.; Petry, W.; Sillescu, H. Z. Phys. B 1993, 91, 357-365.

MA0714870 